\title{
Applying Web Services to define Open Learning Environments
}

\author{
Miguel Ángel Conde, Francisco J. García \\ Computer Science Department. Science Education \\ Research Institute (IUCE). GRIAL Research Group. \\ University of Salamanca \\ e-mail: \{mconde, fgarcia\}@usal.es
}

\author{
María J. Casany, Marc Alier \\ Services \& Information Systems Engineering \\ Department, UPC - Campus Nord, building Omega, \\ Barcelona, Spain. \\ e-mail: mjcasany@1si.upc.edu, marc.alier@upc.edu
}

\begin{abstract}
Evolution of learning processes is almost always linked to social changes and/or technological changes. One of those processes is eLearning, which is involved in the changes brought about by trends 2.0. This new tendencies consider the user, socialization and collaboration as the main important elements and this must be considered also in eLearning contexts. But today learning environments do not facilitate this approach. They are focused on the institutions or courses, not in the student as a key element of the learning process, as well not always provide elements of socialization and collaboration. In order to avoid these drawbacks, and by using service oriented architectures over learning management systems, new elearning environments could be defined. This paper will propose an architecture that would enable learning platforms evolve in that direction.
\end{abstract}

Keywords: PLE, LMS, Web services, Moodle 2.0, eLearning, SOA

\section{INTRODUCTION}

Learning processes are living social elements and therefore they are changing continuously. The evolution of learning processes may be due to new sociological or technological trends, or simply due to pedagogical [1].

The eLearning is one of those evolutions, and is almost completely assimilated into the educational environments. This learning model has relied on technology without obtaining, in many cases, the expected benefit, as if it has occurred in other areas. Despite this eLearning has experienced an extraordinary growth over the last years, learning paradigms; technological solutions, methods and pedagogical approaches have been developed, discarded and adopted. We have reached a point in time when most of learning institutions have adopted the use of Learning Management System (LMS) software, either from commercial vendors or Free Open Source Communities. LMS have reached the balance to meet the structure and (traditional) ways of schools, universities and other educational institutions. These systems provide students and teachers a set of tools for improving learning processes and managing them. However, the LMS have not achieved the expected improvements due to: 1) The tools provided are not used properly and often are used as mere spaces to publish courses [2], 2) The LMS restrict opportunities to collaborate on student learning and promote social constructivism not limited to a period of time (i.e. academic year) [3], 3) They are focused on the course and the institution rather than the student and their needs [4].

Besides this should be noted that online learning does not end with the LMS, but there are plenty of online tools to supplement and improve it.

Therefore, new applications must be taken into account, such as the search applications, news applications, locationenabled applications, content repositories, forums, blogs, calendars, online games, virtual worlds, etc. That is, the new initiatives arising from Web 2.0 [5].

Given this situation will be necessary to evolve the LMS to its integration with contexts that include new technological trends, to provide social characteristics and be focused on the student, these contexts are personalized learning environments (Personal Learning Environments, PLE) [6] and also are considered as Open Learning Environments. This will be the main objective of the present project.

Throughout this article firstly PLE and its relationship with 2.0. tools will be described. Later we talk about a possible deployment by using service-oriented architectures. Finally the proposed architecture will be exposed.

\section{NEW LEARNING CONTEXTS}

LMS are widespread. The learning institutions provide to the teachers and learners a set of online learning tools embedded into the LMS by default. The LMS is also used as a channel to gather other services like access to portfolios and content repositories. But outside of the LMS there are also plenty of online tools that can be useful sources of information, ways of collaborating and sharing: Search, News, Maps, Documents and Spreadsheets, Public forums, Calendar, Translation, Images, Videos, Microblogging. Social networks, Bookmarking, Wave, Online Games, 3d Virtual worlds...

All these services are already being used for educational purposes, but only by advanced users. And usually the usage of these online tools is not integrated within the scope of what we could refer as "virtual classroom". Because is happening outside of the radar of the learning institutions and sometimes even the teacher.

Let's consider this -not so fictional- scenario: a subgroup of students of a class are using tools like Instant Messaging, Wikis, Wave or participating in a Social Networking site, while and sometimes for doing homework. The students engaged in the usage of all these online tools might get better 
grades or not - some studies say they most likely will -, but they will also learn important skills and competences on taking advantage of information technologies to access, share information and collaborate with others to do so. But their teacher may not know about it, because all the information (feedback) he/she receives is what appears on the LMS's course's logs, and all the tools he/she is aware are the ones bundled in the LMS. Sometimes this kind of behavior is empowered by the teacher.

Taking this into account, the integration between LMS and Web 2.0 trends will be very necessary and very important. Every day becomes more essential developing learning to the trends related to Web 2.0. Education must be supplemented by new applications, tools and paradigms that lead to what is called eLearning 2.0 [7]. This new trend in learning requires tools that facilitate: 1 ) changes in the ways of interacting socializing the learning; 2) access to tools considering the new performers in learning, natives and digitals immigrants; 3 ) support to educational trends such as learning throughout life or informal learning, students mobility and so on, related to the Bolonia process; 4) the student-centered learning.

As a proposal to solve all these needs are the PLE, which according to Wilson [6], are defined as learning studentcentered contexts that integrate any tool, service, content, evidence and person involved in e-learning process. This way the responsibility of the learning is given to the student, which benefits itself and its formative process [8]. However, some limits could be necessary (generally derived from an institution concerned about the formality of their teaching/learning process) in the means used by the student to define its learning. Hence it's necessary that PLE can be constructed by integrating tools 2.0 and LMS [9].

Incorporating Web 2.0 applications into learning processes involves bringing in new styles of communication, new roles, new ways of intervention, new scenarios, a wide range of activities, generally, involves opening a series of educational challenges [10]. Through these trends, students are no longer passive subjects of learning to become active students in classes, with no restrictions, being able to use things like Google, Facebook, Twitter, etc. to support learning processes; they can use digital devices such as computers, mobile phones, $\mathrm{mp} 3$, recorders and so on to improve the learning.

The potential of these tools is enormous, as evidenced by experiences such as Jekins [11], Downes [4], and it's increasingly expanding in the different educational environments [12]. Despite all that power is necessary to take into account a number of problems which have arisen through the application of these tools: 1) The improvisation in the use of 2.0 tools and customization of student-centered learning can lead to the wrong idea that the use of this tools into learning should not be planned, this is not correct, they should be estimated, validated and evaluated according to different criteria [13]; 2) Among the problems hampering the full educational utilization of Web 2.0 should be mentioned some technological problems - insufficient bandwidth and lack of access to computers in schools and students' homes and other pedagogical issues where the biggest challenge is that students don't create products and prefer to "copy and learn", students assessment procedures are not formatives and do not "mix" means. [14]; 3) Lack of support from LMS to the use of these kind of tools.

PLE can solve many of these problems and, after determining their use, you must think how to deal with the system definition. Maybe the first question should be whether start a solution from scratch or based it on another LMS. Considering existing initiatives is convenient in this regard, as well as the benefits that would have either decision. In this sense there are some studies and experiences such as [15]:

- Analysis concerning the adequacy of integrating 2.0 applications in continuing training context, as well as in web application hybrid (mash-ups).

- Definition of PLE from an hybrid approach, describing a customizable Web portal as a base of PLE in which would be aggregated different Web tools interconnected from different contexts, and providing an interaction language among its users.

- Research of the limitations of the integration of applications derived from the exchange of information between them, the use of REST is proposed to solve them.

- Design and implementation of strategies for PLEs definition.

- Implementation of a institutional PLE for the University of the Basque Country, thus, a PLE where applications 2.0 which student can use are defined by the University.

- RWTH Aachen University was commissioned to develop the Learning Environment Framework (PLEF) (http://eiche.informatik.rwthaachen.de:3333/PLEF/index.jsp), a PLE service that takes care of composing a set of subservices to support the students' activity when composing, manage, tag, annotate and share their favorite resources.

The discussed initiatives define in some cases PLE, but do not consider all areas of information. Most of them only integrate different tools 2.0 in a container, regardless of LMS information. One of them, the Basque Country University works to integrate tools in the LMS, but this integration will be through widgets and will not exist, therefore, interaction between tools and platform.

Hence, the system proposed here covers a broader spectrum of communication and do not forget in any case the LMS. Taking into account these experiences could be decided that it might be better to hold the system from scratch, taking the architecture referred to the mash-ups and the interaction languages, but should be necessary to consider the futility of reinventing the wheel again. To define a PLE should not assume to discard the functionalities of the LMS, these functionalities can enrich student learning, and 2.0 components integrated in the learning platform would enhance exponentially the possibilities of learning in institutions. Neither should be neglected the acceptance of certain LMS such as Moodle in Spain (and elsewhere in the world). 
Once the base has been established, the conversion of LMS into a PLE begins. At this point, the two possibilities of PLE that this project offers should be considered. First, the need to export information from the LMS to a PLE, i.e., the export system of educational portable components, while on the other hand would be the integration of tools 2.0 in the LMS in order to redefine them as PPLE (integrated PLE or institutional PLE, depending on the approach).

Insomuch as the export of information of the platform, you must determine what to import, how to import it and how to display the information.

With regard to what to export, pedagogical criteria must be met considering what information is most critical, which activities are more representatives and enrich the personal student environment, in addition to what information is more appreciated by the students.

Regarding to how to export is considered the usage of a service-oriented architecture (SOA - Service Oriented Architecture). It allows, so, the integration of the system regardless of the technology that is implemented, the scalability is improved, more flexible systems are defined, easier to maintain and more resistant to change. It also facilitates the interconnection of heterogeneous contexts such as LMS and mobile devices.

\section{ARCHITECTURE PROPOSAL}

There is currently a trend towards modularization of computing systems. This modularization is due to the advantages that it entails, such as independence of development, increase in security, scalability, etc. Moreover, work is being carried out towards the production of software services independent of the underlying implementation. The result of merging both ideas is Service Oriented Architectures (SOA). Among the elements that favored the development of SOA are the developments of different types of applications, computer networks, client-server architectures, etc. [16]. SOA implies a step further in the development of information systems architecture. In its most basic form, SOA is a set of services that communicate with one another [17].

In educational contexts, the application of SOA will be useful in order to adapt the current LMS to emerging technologies, frameworks and specifications and, this way, to transform these legacy systems into service-based eLearning platforms [18]. It is clear, therefore, that the application of these architectures enables communication with learning platforms. Accordingly, there are some initiatives of application of SOA to learning platforms with different purposes, among which we could mention the following:

- Adaptation of part of an LMS services seeking mobility [19].

- Definition of SOA to information recovery and search based on semantic contents [20].

- Integration of learning tools in other systems [21].

- X. Qiu and A. Jooloor [22], proposes that the advantages shown in extending the Moodle to use WSMS can be applied generically to all LMS.

Regarding the LMS we have selected (Moodle), the groups that propose this project (GESSI, from the
Polytechnic University of Catalonia and GRIAL, from the University of Salamanca) are working together towards the integration of a SOA in this LMS. To adapt Moodle into SOA is not an easy task, as it requires a profound knowledge of the main libraries in Moodle, the functionalities available, the capabilities of each user, etc.

In 2008, the GESSI group was entrusted by Martin Dougiamas, founder of Moodle, with the development of a new API to access the services of the Moodle kernel regardless of its implementation, which would remain stable in future, Moodle implementations. This API consists of a set of web services, where most functionalities an external application might need are encapsulated. In October, 2008, the web service layer was integrated in some Moodle distributions for testing purposes. This layer aims at being useful for all developers who wish to define applications for Moodle without the need to touch the LMS code.

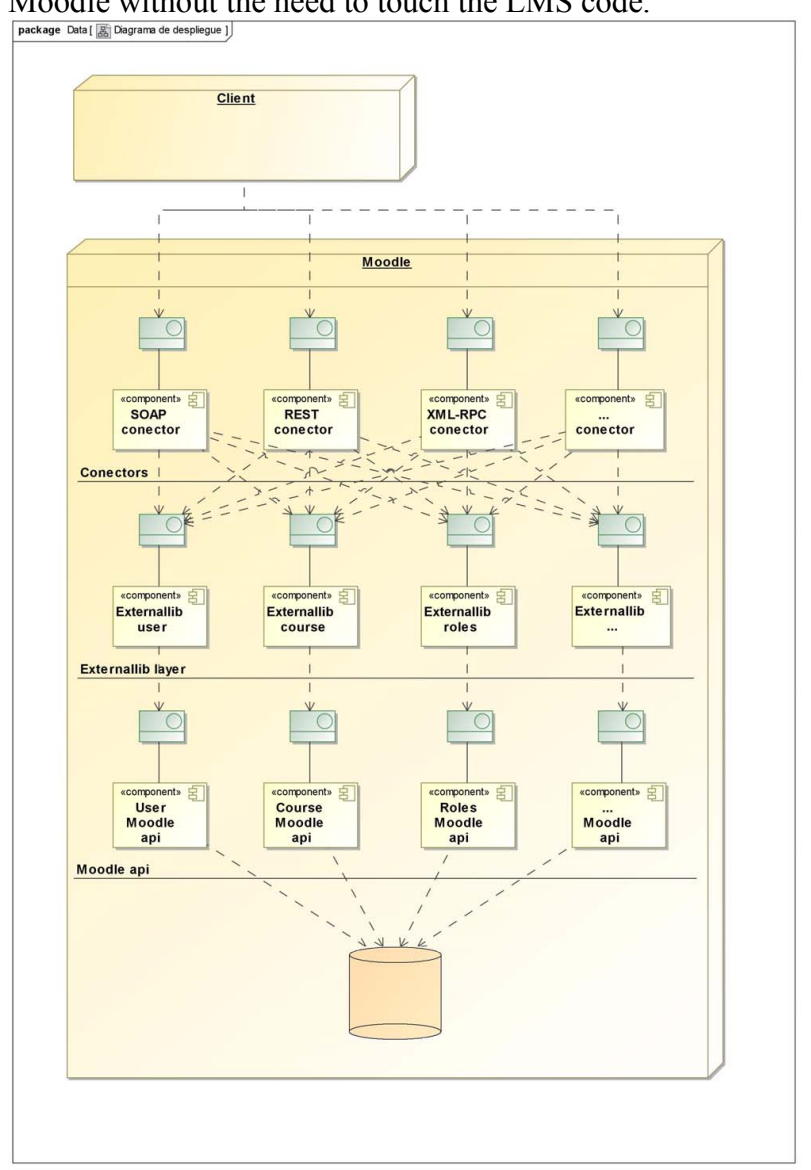

Figure 1. Moodle Web services Architecture

This API will become, in the present project, the base for the development of a Service Oriented Architecture consisting of (Figure 1.): 1) a scalable layer of connectors that will keep separated the communication protocol applications from the web services and that will allow the addition of new protocols that might appear in the future; 2) an integration layer that serves as an access point for the functionalities of Moodle, this layer interacts with Moodle 
API 3) a layer which groups Moodle kernel functionalities and provides interfaces to facilitate communication between Moodle and the other layers.

Some of the main services developed for Moodle are shown in the Figure 2, described using SOAml.

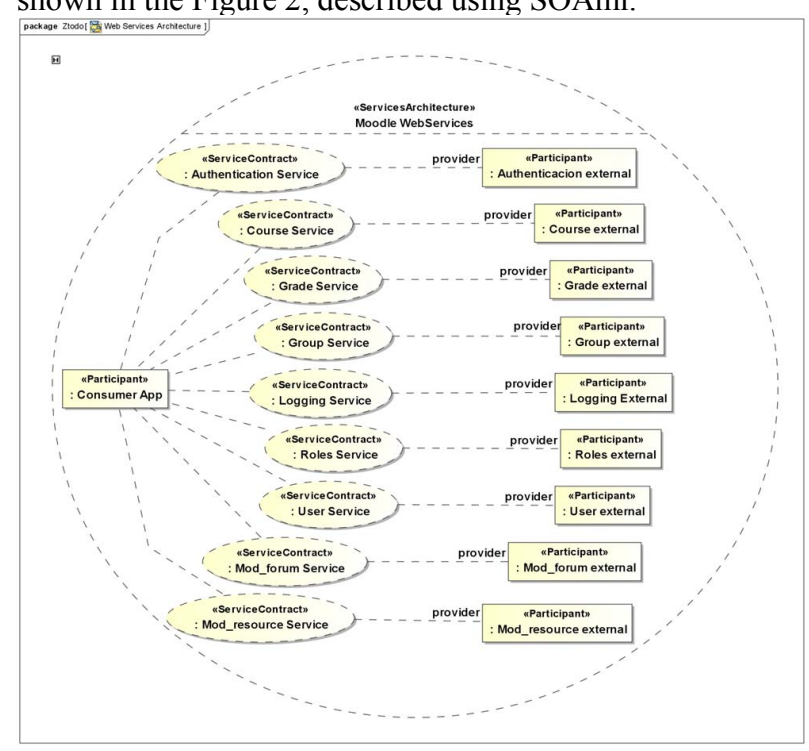

Figure 2. Some of web services Moodle contracts.

Some applications of this architecture could be seen in [23], such as a backoffice application or a system which allows Moodle logs visualization.

The architecture will be responsible for providing information, but it has to be considered how to visualize this information and how to integrate it as a portable educational element, in this case, as a widget. Widgets are small and portable elements that can be run in any HTML context [24], providing functionality "live", content or functionality from some other website. Therefore, information from different 2.0 applications could be shown, as well as information present in an LMS. As far as the application of the project is concerned, a system capable of generating widgets out of information from Moodle would have to be implemented. To make these widgets easy to integrate in different containers, different types of widgets must be considered.

Therefore, the widget generation model has to be defined following the information in Moodle as the source of portable educational components for their integration in PLEs external to the LMS.

Another possibility to take into account is the idea of integrating external tools into Moodle with the aim of providing students with a PLE within Moodle. For each of these tools, careful attention should be paid to how they should be implemented and how they should be presented to students and teachers so that they could find them useful. Also, must be determined the type of feedback that would be useful both for the teacher and for the institution or, in other words, how the integrated tool would interact with Moodle.

\section{NEW APPROACH TOWARDS PERSONAL LEARNING ENVIRONMENTS}

Given the above, it will pose an architecture that exploits the SOA layer component of Moodle. Specifically, the external layer we will used (a layer which access to internal Moodle information), also a set of web services will be defined, geared to the specific needs of the types of environments to define, and connectors will be the link to other systems. On that basis, the architecture will incorporate an educational component model that enables portable platform information and export it to contexts 2.0. This capability allows users to construct their knowledge, but also must be considered to control the process. That is why we consider the possibility of incorporating 2.0 tools within the LMS. To do this, an IMS LTI gateway will be used, allowing that external applications could be included transparently into Moodle.

Figure 3 shows the distribution of the different architectural components. Gray color shows the SOA components as well as a set of connectors to facilitate access to it. An engine to define learning portable components will use those connectors, and those components will compose the PLE (red). The widget engine will generate Learning Portable Components in different widgets technologies, through the information extracted from Moodle. The new widgets combined with 2.0 Tools will result in the PLE. The current proposal also includes the integration of 2.0 tools into Moodle by using a gateway based on IMS-LTI.

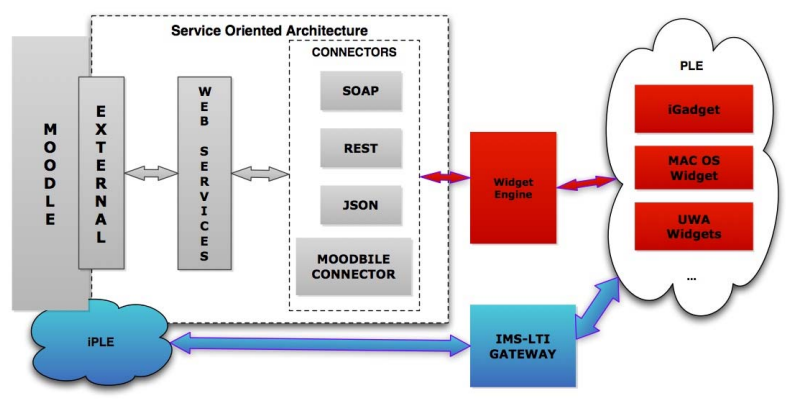

Figure 3. Architecture proposal

\section{CONCLUSIONS}

eLearning needs evolve, and this evolution must consider the student. Usually, in online learning processes, the student is conditioned to temporal issues, LMS technical constraints or an inefficient use of learning platforms features. If we don't consider the students as the center of elearning processes, if new technological and sociological trends are not considered, any elearning activity will fail.

To avoid this situation will be necessary the personalization of LMS and its integration with 2.0 tools and applications. This is the main reason of the definition of PLE and our proposal considers the two possible ways of its application. Free personalization leaded by the student and the possibility of personal learning environments conducted by an organization. These two new learning practices must be supported by technologies, and must consider existent 
Learning Management Systems such as Moodle. This platform includes a web service layer that allows the connection with other application and establishes a medium of exchange of information, which could be used to define learning portable components that will compound new personalized learning contexts.

This project is a starting point for new eLearning initiatives such as integration of 2.0 tools in the LMS, the definition of personalized learning environments, content exportation to new areas like mobile devices and so on.

\section{ACKNOWLEDGMENT}

This work was supported by the Spanish Industry Ministry (project TSI-020302-2009-35) and by the Castile and Lion Regional Government through GR47 excellence project

\section{REFERENCES}

[1] F. J. García Peñalvo, "Estado Actual de los Sistemas E-Learning," Teoría de la Educación. Educación y Cultura en la Sociedad de la Información, vol. 6, no. 2, Octubre 2005, 2005.

[2] U. o. N. Carolina, "Sakai Pilot Evaluation Final Report,"

http://www.unc.edu/sakaipilot/evaluation/FinalRep t-Oct15-09-sm.pdf, [29/01/2010, 2009].

[3] J. S. Brown, and R. P. Adler, "Minds on Fire: Open Education, the Long Tail, and Learning 2.0.," Educause Quarterly, vol. 42, no. 6, pp. 16-32, 2008.

[4] S. Downes, "E-learning 2.0," Elearn magazine, http://elearnmag.org/subpage.cfm? section=articles \&article=29-1, [29/01/2010, 2006].

[5] T. O'Reilly, "What is Web 2.0: Design Patterns and Business Models for the Next Generation of Software," http://oreilly.com/web2/archive/whatis-web-20.html, [29/01/2010, 2005].

[6] S. Wilson, O. Liber, M. Johnson et al., "Personal Learning Environments: Challenging the dominant design of educational systems "Journal of $e$ Learning and Knowledge Society, vol. 3, no. 3, pp. 27-38, 2007.

[7] H. Ajjan, and R. Hartshorne, "Investigating faculty decisions to adopt Web 2.0 technologies: Theory and Empirical Tests," The Internet and Higher Education, vol. 11, no. 2, pp. 71-80, 2008.

[8] M. R. Lepper, "Microcomputers in education: Motivational and social issues.," American Psychologist, vol. 40, pp. 1-18, 1980.

[9] A. Gogoulou, E. Gouli, M. Grigoriadou et al., "A Web-based Educational Setting Supporting Individualized Learning, Collaborative Learning and Assessment," Educational Technology \& Society, vol. 10, no. 4, pp. 242-256, 2007.

[10] SCOPEO, "Formación Web 2.0," Monográfico SCOPEO, http://scopeo.usal.es/images/documentoscopeo/sco peom001.pdf, [29/01/2010, 2009].

[11] H. Jenkins, Convergence Culture: Where Old and New Media Collide, New York: NYU Press, 2006.

[12] J. De Pablos, "El cambio metodológico en el espacio europeo de educación superior y el papel de las tecnologías de la información y la comunicación," Revista Iberoamericana de Educación a Distancia, vol. 10, no. 2, pp. 15-44, 2007.

[13] C. Suárez, Educación y virtualidad: URP, 2008.

[14] BECTA, "Web 2.0 technologies for learning at KS3 and KS4 - Project overview," http://partners.becta.org.uk/index.php?section=rh\& catcode $=$ re rp 02\&rid $=14543, \quad[29 / 01 / 2010$, 2008].

[15] O. Casquero, J. Portillo, R. Ovelar et al., "PLE Network: an integrated eLearning 2.0 architecture from University's perspective," Interactive Learning Environments, (in Press).

[16] R. Ramaratnam, "An analysis of service oriented architectures.," System Design and Management Program,

http://dspace.mit.edu/bitstream/handle/1721.1/4237 2/234382950.pdf? sequence $=1$, [29/01/2010, 2007].

[17] B. R. Payne, and A. J. Barrody, Service oriented architecture. , Rochester Institute of Technology, 2006.

[18] D. Dagger, A. O'Connor, S. Lawless et al., "Service-Oriented E-Learning Platforms: From Monolithic Systems to Flexible Services," Internet Computing, IEEE, vol. 11, no. 3, pp. 28-35, 2007.

[19] S. Kurz, M. Podwyszynski, and A. Schwab, $A$ Dynamically Extensible, Service-Based Infrastructure for Mobile Applications, 2008.

[20] LUISA, "Learning Content Management System Using Innovative Semantic Web Services Architecture. ," http://luisa.atosorigin.es, [29/01/2010, 2009].

[21] S. Pätzold, S. Rathmayer, and S. Graf, "Proposal for the Design and Implementation of a Modern System Architecture and integration infrastructure in context of e-learning and exchange of relevant data," ILearning Forum 2008., E. I. F. E-Learning, ed., 2008, pp. 82-90.

[22] X. Qiu, and A. Jooloor, "Web Service Architecture for e-Learning," Journal of Systemics, Cybernetics and Informatics, vol. 3, no. 5, pp. 92-101, 2006.

[23] M. A. Conde, D. A. Gómez, A. Del Pozo et al., "Moodle 2.0 Web Services Layer and Its New Application Contexts," Communications in Computer and Information Science. pp. 110-116.

[24] W3C, "Widgets 1.0 Packaging and Configuration," http://www.w3.org/TR/widgets/, [29/01/2010, 2008]. 